Cardiology in the Young (2016), 26, 1039-1043

doi: $10.1017 /$ S1047951115002553
(C) The article has been co-published with permission in HeartRhythm and Cardiology in the Young. All rights reserved in respect of Cardiology in the Young. (c) The Author 2016. For HeartRhythm, (c) The Author 2016.

\title{
Pediatric \& Congenital Electrophysiology Society: building an international paediatric electrophysiology organisation
}

\author{
Mitchell Cohen, MD, CEPS-PC, FACC, FHRS, ${ }^{1}$ Shubhayan Sanatani, MD, FHRS, ${ }^{2}$ \\ Elizabeth Stephenson, MD, MSc, CEPS-PC, FHRS, ${ }^{3}$ Jon Skinner, MbChB, FRACP, MD, ${ }^{4}$ Fabrizio Drago, MD, \\ Andrew Davis, MB BS, MD, FHRS, CEPS-PC, ${ }^{6}$ Jan Janousek, MD, ${ }^{7}$ Eric Rosenthal, MD, FRCP, MRCPCH, ${ }^{8}$ \\ Kathryn K. Collins, MD, FHRS, ${ }^{9}$ John Triedman, MD, FACC, FHRS ${ }^{10}$ \\ ${ }^{1}$ Heart Center Phoenix Children's Hospital and University of Arizona College of Medicine-Phoenix, Phoenix, Arizona, \\ United States of America; ${ }^{2}$ Division of Pediatric Cardiology, British Columbia Children's Hospital, Vancouver, British \\ Columbia, Canada; ${ }^{3}$ Department of Pediatrics, University of Toronto, Labatt Family Heart Centre, The Hospital for Sick \\ Children, Toronto, Ontario, Canada; ${ }^{4}$ Starship Children's Hospital, Child and Health Auckland University, Auckland, \\ New Zealand; ${ }^{5}$ Pediatric Arrbythmias Complex Unit, Pediatric Cardiology and Cardiosurgery Department, Bambino \\ Gesù Pediatric Hospital, Rome, Italy; ${ }^{6}$ Royal Children's Hospital, Melbourne, Australia; ${ }^{7}$ Children's Heart Centre, \\ Motol Children's Hospital, Prague, Czech Republic; ${ }^{8}$ Evelina Children's Hospital and Guy's and St. Thomas NHS' \\ Trust, London, United Kingdom; ${ }^{9}$ Arrbythmia Center Children's Hospital Colorado, Denver, Colorado; ${ }^{10}$ Boston \\ Children's Hospital and Harvard Medical School, Boston, Massachusetts, United States of America
}

\begin{abstract}
The Pediatric and Congenital Electrophysiology Society (PACES) is a non-profit organisation comprised of individuals dedicated to improving the care of children and young adults with cardiac rhythm disturbances. Although PACES is a predominantly North American-centric organisation, international members have been a part of PACES for the last two decades. This year, PACES expanded its North American framework into a broadly expansive international role. On 12 May, 2015, paediatric electrophysiology leaders from within the United States of America and Canada met with over 30 international paediatric electrophysiologists from 17 countries and five continents discussing measures to (1) expand PACES' global vision, (2) address ongoing challenges such as limited resource allocation that may be present in developing countries, (3) expand PACES' governance to include international representation, (4) promote joint international sessions at future paediatric EP meetings, and (5) facilitate a global multi-centre research consortium. This meeting marked the inception of a formal international collaborative spirit in PACES. This editorial addresses some solutions to breakdown the continental silos paediatric electrophysiologists have practiced within; however, there remain ongoing limitations, and future discussions will be needed to continue to move the PACES global international vision forward.
\end{abstract}

Keywords: Electrophysiology; international collaboration; paediatrics

Received: 18 October 2015; Accepted: 19 October 2015; First published online: 14 April 2016

$\mathrm{P}$ AEDIATRIC ELECTROPHYSIOLOGY IS A UNIQUe discipline within paediatric cardiology, bridging a traditional outpatient clinic and inpatient and intensive care consulting role with an interventional

Correspondence to: Dr M. Cohen, MD, FACC, FHRS, Co-Director of the Heart Center and Chief, Pediatric Cardiology at Phoenix Children's Hospital, Vice-President of Pediatric and Congenital Electrophysiology Society (PACES), 2nd Floor Heart Center, 1919 East Thomas Road, Phoenix, AZ 85016, United States of America. Tel: 602933 3366; Fax: 602-933-4166; E-mail: mitchcohenmd@gmail.com procedure-based model. Paediatric electrophysiologists care for children and adults with CHD who have arrhythmias using a combination of pharmacological and non-pharmacological therapies, including implantable devices and catheter ablation. In addition, with the expanding role of genomics in cardiovascular medicine, paediatric electrophysiologists have become the "go-to" experts for channelopathies such as long QT syndrome, Brugada syndrome, and 
catecholaminergic polymorphic ventricular tachycardia. Sudden death in the young has received increasing attention in both medical and lay media, and paediatric electrophysiologists have the expertise to guide the field, including screening programmes, advocacy, and risk stratification for sudden death in children with CHD and cardiomyopathies. In this respect, paediatric electrophysiologists are often the central core of a multi-disciplinary team. Most large children's hospitals in North America have 2 or more paediatric electrophysiologists; however, on an international level, most other countries rarely have more than 1 paediatric electrophysiologist - who may function additionally in an interventional or general role - per centre, whereas in some cases there may only be 1 paediatric electrophysiologist in a entire country, caring for a large number of children spread across vast regions. Other countries do not have any providers specifically trained in paediatric electrophysiology, but one or more adult electrophysiologists who work together with paediatric cardiologists to provide care.

As the world has become smaller with web-based communication, it has become progressively easier for paediatric electrophysiologists to communicate across previously challenging barriers. Individually, many Pediatric and Congenital Electrophysiology Society (PACES) members have reached out internationally to establish collegial professional relationships with practitioners in other countries, based on formal and informal visits and exchanges; however, effective implementation of a global paediatric electrophysiology community also requires an organising body that oversees and facilitates this activity - a body which nearly all paediatric electrophysiologists view as their "home". To address this need, PACES recognised its responsibility in opening a dialogue beyond its traditional North American framework into a broadly expansive international role. It is possible that such an undertaking may be considered worthwhile by other paediatric cardiology subspecialty medical societies.

\section{PACES as an organisation}

Founded by clinician leaders, PACES is a non-profit organisation comprised of individuals dedicated to improving the care of children and young adults with cardiac rhythm disturbances; one of the society's missions is to foster high-quality collaboration and research and exchange of ideas on arrhythmia topics that are relevant to the interests of children and young adults with CHD. PACES is the "community of practice" to the Heart Rhythm Society with expertise in congenital and paediatric electrophysiology.
Membership in PACES consists of four types: (1) Regular, (2) Associated Professional, (3) Trainee, and (4) Affiliate. Regular membership is open to individuals with an $\mathrm{MD}, \mathrm{DO}$, or $\mathrm{PhD}$ degree who are interested in arrhythmias in the paediatric and CHD patient, who have completed training, and who are engaged in the practice of Paediatric Cardiac Electrophysiology through clinical service, research, or teaching. Associated professional membership is open to individuals who are interested in arrhythmias in the paediatric and CHD patient, assist in paediatric cardiac electrophysiology through clinical support, research, or teaching, and do not hold one of the degrees required for regular membership. Regular and associated professional members are eligible to vote, hold office, and serve on committees. The elected officers of the society are the President, Vice President for Administration, Vice President for Finance, and Vice President for Research and an Associated Professional Representative. These officers and the two immediate past presidents comprise the Executive Committee. In addition, there are six standing committees: (1) Guideline Development, (2) Guideline Review, (3) Advocacy, (4) Finance, (5) Training, and (6) Research. These committees are chaired by a senior electrophysiologist and have an additional $10-15$ physician and advanced practice nurses allowing for greater membership participation.

\section{International paediatric electrophysiology meetings}

Although PACES began in the 1980s with only a very small group of paediatric electrophysiologists, it now claims over 400 members worldwide. Although the small size of the society presents certain challenges, it also provides tremendous opportunities for innovation and growth, and one such opportunity is the expansion of our international presence, both in numbers of members and in the development of a shared global vision. On May 12, 2015, the predominantly North American leadership of PACES met with over 30 international paediatric electrophysiologists representing 17 countries - Australia, Canada, Chile, China, Costa Rica, Czech Republic, Finland, Germany, Hungary, Israel, Italy, Japan, New Zealand, South Korea, United Kingdom, United States of America, and Vietnam.

Before this inaugural meeting, a list of discussion topics was sent to all invited representatives to serve as a framework of possible items of interest for both North American and international representatives. These items included the following:

- Collaborative clinical scientific research

- Development of practice guidelines 
- Clinical and research training and academic exchanges

- Global quality improvement collaboration

- Sharing of globally relevant best practices

- Effect of healthcare systems and finance on access to technology

- Cost competition, international referrals, and selfreferrals in a globalised world

- Expansion of established paediatric electrophysiology programmes to international satellite organisations

- Second opinions and case review

- Medical missions

\section{Expanding PACES' global vision: opportunities}

After extensive discussions by all participants around many of the areas listed above, it became quite apparent that a more inclusive global paediatric electrophysiology vision was initially going to require four changes within the current PACES model.

\section{Inclusion of international representation in PACES governance}

All international members expressed a strong opinion that an international representative be added to the Executive Committee. To this end, the organisation's by-laws have been amended to add an International Executive Committee Member voted on by all PACES members. Individuals eligible for this position should have his/her clinical practice outside of North America. The goal of the International Executive Committee Member will be to help facilitate and foster continued growth of PACES at a global level. It was decided at this meeting that this individual would work with North American and international members of the Advocacy Committee to promote global quality initiative programmes. This individual will be part of the PACES leadership creating an international dimension and building a worldwide network among paediatric electrophysiologists outside of North America.

\section{Joint paediatric and congenital sessions at annual international meetings}

Before 2015, there were traditionally three large annual paediatric electrophysiology society meetings worldwide: (1) North American Heart Rhythm Society, (2) Asia-Pacific Heart Rhythm Society, and (3) European Heart Rhythm Society. Paediatric and congenital electrophysiology has traditionally occupied small subsections of the meetings, and joint societal symposiums have rarely been organised. To foster and enhance international academic collaboration, each of the societies has recently established a formal joint session focussed on paediatric and congenital topics. The Association of European Pediatric Cardiology in conjunction with PACES held a formal joint symposium in Prague (May, 2015) on "Primary Prevention of Sudden Cardiac Death in Paediatric and CHD Patients". Having successfully held a similar joint session at the 2014 Asia-Pacific Heart Rhythm Society in New Delhi, India, the Asia-Pacific Heart Rhythm Society Scientific Sessions in Melbourne, Australia, held a joint session with PACES in November 2015 entitled "Answering the Challenges from Pediatric Electrophysiologists in the Asia-Pacific Region". In addition, in May, 2016, at the Heart Rhythm Society's 37th Annual Scientific Sessions in San Francisco, there will be a joint session with representatives of PACES and Association of European Pediatric Cardiology. These learning sessions are focussed to meet the needs of international audiences and are an excellent opportunity for attendees to learn about novel treatment strategies for arrhythmic conditions outside of their primary geographical area of practice, as certain anti-arrhythmic agents, devices, or catheters may have been released in a particular country before more global availability. At the same time, visiting speakers will have opportunities to learn how other leaders in different working environments cope with their unique challenges. Electrophysiology trainees will also benefit enormously from these sessions, which may challenge their didactic training programmes and core assumptions.

\section{International input for development and harmonisation of practice guidelines}

A robust discussion focussed on the development of a more internationally inclusive policy around the development and adoption of practice guidelines was needed. Over the last 5 years, PACES has established a formal process by which all documents requiring paediatric electrophysiology endorsement are reviewed by our Guideline Review Committee. This committee has a 3-year rotating membership term, and now includes international members. In doing so, PACES hopes to minimise both redundancy and disparity among scientific guidelines that may be developed in multiple societies. PACES-initiated documents and guidelines now request reciprocal paediatric endorsement from members of guideline committees of other paediatric electrophysiology societies such as Asia-Pacific Heart Rhythm Society or Association of European Pediatric Cardiology - for example, the upcoming PACES-led Pediatric Ablation Consensus Statement was endorsed by the Association of European Pediatric Cardiology. 


\section{Facilitation of global multi-centre research}

A unique aspect of PACES is our formal research policy. PACES strongly encourages researchers who are developing multi-centre studies and seeking PACES endorsement to submit their research projects to the Research Committee for review. The Research Committee has international representation and can support initiating investigators to develop broad appeal to membership; however, it has been suggested that, to date, many PACES-endorsed research projects have been "North America-centric" and not easily conducive to international participation. A significant role of the newly elected International EC Member will be to work with members of the Research Committee to foster international participation in PACES-endorsed research projects as well as to become local principal investigators in these projects. To facilitate a global research mission, the PACES website has been rebuilt to allow sharing of Research Ethics and consent forms and protocol and enrolment updates through a secure portal.

\section{Expanding PACES' global vision: ongoing challenges}

\section{Practice variation among developing countries and isolated practitioners}

It is the intent of PACES to welcome all those who share the PACES vision. In many countries, limitations of medical resources are one of the most challenging problems related to caring for children with arrhythmias. How can a single paediatric electrophysiologist in Vietnam care for a population of over 80 million in the same manner that more than 20 Canadian paediatric electrophysiologists care for a population less than half the size? Although the population within countries represented at the meeting ranged from 4.4 million to 1.4 billion, the number of paediatric electrophysiologists practicing relates less to the population than to the national gross domestic product (Table 1$)^{1}$

Addressing these issues of global disparity will require an appreciation for the challenges facing practitioners in areas that are less able to devote medical resources to child healthcare and congenital paediatric electrophysiology. This will require openness to regionally appropriate global variations in practice and create high-value solutions to the high cost of arrhythmia care. The daily challenges faced by a solo paediatric electrophysiologist serving a massive population in a developing country are vastly different from those of an academic centre. This disparity is one of the principal challenges facing paediatric electrophysiologists interested in extending the global reach of this subspecialty, one that simple training of additional international paediatric electrophysiologies will not completely address. The needs of our patient group may be met by individuals or teams, which may bear little resemblance to those of a typical North American model; however, as countries like Vietnam, India, and China develop their healthcare services, practitioners in our specialty may seek guidance in establishing services, advice with difficult cases, and opportunities for practical hands-on mentoring. It is within this realm that PACES aims to extend its global impact - for example, PACES has the

Table 1. Number of paediatric electrophysiologists per capita GDP.

\begin{tabular}{lccc}
\hline Country & Population & GDP* & Estimate of paediatric EP (MD/DO) \\
\hline Argentina & $41.45 \mathrm{M}$ & 5 & 5 \\
Australia & $23.13 \mathrm{M}$ & 14,715 & 51,958 \\
Canada & $35.34 \mathrm{M}$ & 51,958 & 3 \\
Chile & $17.62 \mathrm{M}$ & 15,732 & Unknown \\
China & $1.4 \mathrm{~B}$ & 6807 & 1 \\
Costa Rica & $4.87 \mathrm{M}$ & 10,184 & 3 \\
Czech Republic & $10.52 \mathrm{M}$ & 19,844 & 1 \\
Finland & $5.43 \mathrm{M}$ & 27,927 & 1 \\
Germany & $80.62 \mathrm{M}$ & 31,252 & 2 \\
Hungary & $9.89 \mathrm{M}$ & 13,480 & 7 \\
Israel & $8.06 \mathrm{M}$ & 36,051 & 5 \\
Italy & $59.83 \mathrm{M}$ & 35,925 & 1 \\
Japan & $127.3 \mathrm{M}$ & 38,633 & Unknown \\
New Zealand & $4.4 \mathrm{M}$ & 41,555 & 2 \\
South Korea & $50.22 \mathrm{M}$ & 25,976 & 14 \\
Spain & $46.77 \mathrm{M}$ & 29,863 & 1 \\
United Kingdom & $64.1 \mathrm{M}$ & 41,787 & 1 \\
United States of America & $318.9 \mathrm{M}$ & 53,041 & 1910 \\
Vietnam & $89.71 \mathrm{M}$ & & 240 \\
\hline
\end{tabular}

$\mathrm{EP}=$ paediatric electrophysiology

*GDP is based on 2013-2014 data 
capacity to identify experts and facilitate collegial discussion and second opinions in challenging areas such as cardiac channelopathy, arrhythmic conditions related to adult congenital disease, and complex management problems encountered in ablation and device therapy. In addition, partnership programmes for training and clinical support should be developed.

\section{Adult CHD and inherited disorders}

The rhythm management of the older child and adult with repaired CHD requires a team with a unique skill set. The locus of care of the adolescent patient and adult with CHD in the United States of America and abroad is exceedingly heterogeneous. In most highvolume North American paediatric electrophysiology programmes, paediatric electrophysiologists are the sole providers of paediatric electrophysiology care to teenage and, in many cases, adult congenital populations; however, in other countries where paediatric electrophysiology services may presently be geographically limited to a small number of academic centres - for example, India and China - the care of the adolescent often falls to the adult electrophysiologist. Other hybrid models of paediatric and adult paediatric electrophysiology collaboration are also successfully employed around the world. Furthermore, partnerships such as this are imperative when it comes to the management of families with inherited arrhythmic syndromes. Expertise in cardiac ion channelopathies and cardiomyopathies may cross disciplines, including relationship with clinical and molecular geneticists, genetic counsellors, and forensic pathologists. It was beyond the scope of this international meeting to address the complex question "Who is best to care for the adolescent and older congenital patient?". The discussion about how these teams are best formed, trained, and accredited will develop and there will be international variance. It is important to continue to bring this question to the forefront, seeking flexible policies that are relevant to a wide range of social and medical conditions, and respectful of the unique medical and psychosocial needs of adolescents and adult congenital patients.

\section{Conclusions}

The mission of PACES is to promote collaboration and research among those caring for children and young adults with arrhythmic conditions. Global implementation of this mission will be challenging. In 2015, PACES leadership, which historically has been predominantly North American, met with over 30 international paediatric electrophysiology representatives from over 17 countries to discuss both opportunities and challenges that exist in formalising international collaboration. Discussed among the attendees were measures to add an international member to the executive committee, formalise joint societal symposiums at national meetings, and collectively review and endorse scientific statements from external societies. The attendees recognised that vast differences across countries exist as it relates to resource allocation in caring for children with complex arrhythmias. These differences pose a unique set of challenges and opportunities. Expansive use of the web may allow for second opinions to be performed and facilitate best practice models. It is the goal of PACES that this meeting constitutes the formal inception of a collaborative effort among paediatric electrophysiologists from North America, South America, Europe, and Asia-Pacific regions to work together in areas of advocacy, research, and guideline development.

\section{Reference}

1. GDP per capita (current US\$) | Data I Table - The World Bank [Web log post]. Retrieved August 1, 2015 from http://data. worldbank.org/indicator/NY.GDP 